\title{
Observations of Microalbuminuria and its Association with Cardio- Vascular Complication in Type 2 Diabetes
}

\author{
Authors \\ Dr Anand Kumar ${ }^{1}$, Dr Pankaj Hans ${ }^{2}$ \\ ${ }^{1}$ Senior Resident Depart. Of Medicine, PMCH Bihar \\ ${ }^{2}$ Associate Prof. Depart. Of Medicine, PMCH Bihar
}

\section{Introduction}

Diabetes mellitus is the commonest endocrine disease found in general population, as such diabetes mellitus is a syndrome (a collection of disorders of which hyperglycemia is the hallmark).

The disease is known from ancient time, Charka and Sushruta (600-400BC) of India recognized the disease and named it "Madhumeha" (rain of honey), having noted the sweetness of urine. The names diabetes (Siphon) was given by Aretaeus in the first century AD increase in blood sugar as a cardinal sign was first noted by Claude Bernard (1950).

The incidence for diabetes mellitus is much more common in India than Western counterpart hence data published on western population are not directly applicable to our own population. About $98 \%$ of our diabetic population belongs to NIDDM. The prevalence of NIDDM was first survey on the township in Kudermukh. In this urban population diabetes was present in $5 \%$ of people aged 20 years. The peak prevalence $(41 \%)$ was in the group aged 55-64 years. This result further became stronger when Madras survey result was published. The age adjusted prevalence of diabetes was $8.2 \%$ in the urban and $2.4 \%$ in rural population in Madras. Due to high incidence of diabetes in Indian it became like an epidemic disease. Because of this importance of NIDDM in Indian population we choose it for our study.

\section{Microalbuminuria}

Diabetic patients who develop proteinuria show a marked increase in cardiovascular morbidity and mortality. (Relative risk 7.40, 95\% CI 2.04-7.62) and CHD mortality (relative risk 7.40, 95\% CI 2.94-18.7) when adjusted to age only. Its independent predictions power did not persist. However, in age adjusted multivariable survival analyzing that allowed for other significant risk factors. Male sex, pre existing CHD, high levels of glycated $\mathrm{Hb}$, and high serum cholesterol. The precise pathogenesis of human diabetic kidney disease and the factors responsible for the susceptibility to it remains in past, obscure. However, this is now evidence the renal disease cluster in families and that genetic factors may be of central importance in determining susceptibility. Predisposition of attend hypertension has been suggested as playing a contributory role in the development of kidney disease. Hypertrophic process may be implicated 
in the susceptibility of arterial was damage and glomerular injury in diabetes.

The first clinical involvement is the appearance of microalbumin and a small elevation in arterial pressure. Mesangial expansion accompanies these changes microalbuminuria is associated with abnormalities of lipoprotein profiles. The environment changes brought about by diabetes could lead in susceptible individuals to increased systemic and intraglomerular pressure on the other hand. These two processes would cause proteinuria and glomerulosclerosis.

In the early part of changes there is hyper filtration and latter on there is hypertrophy of kidney. In this time there is hypertrophy of kidney. In this time there is microalbuminuria. Albumin in the urine not diagnosed by usual albustix but only by radioimmunoassay, turbimetry of micralclip stick test.

An expert group has recently declined microalbuminuria as urine albumin excertion rate between 30 and $300 \mathrm{ug} / \mathrm{min}$ in an overnight or 24 hours sample on at least 2 of 3 occasions. Within a period of six months, an albumin excertion rate $20-200 \mathrm{mg} / \mathrm{min}$ is approxmiately $30-300 \mathrm{mg} / 24$ hours or $3-30 \mathrm{mg} / \mathrm{molcreatinine.}$

The frequency of microalbuminuria are differs in different report. in one report frequence of microalbuminuria in type II diabetes is 20-305 but sehnack found a prevalence of 59\% Mattock at al (26\%) Kikhava at al 21\% Microalbuminuria are found more in male female.

The risk factors associated with CHD are present in microalnumiuric diabetic patients. in NIDDM population the systolic blood pressure is higher in comparison to normoalbuminuric patients (Aschmitz, Vaeth M, thokild Faris et al) in lipid abnormality,triglycerdemia is increased and also post prandial triglycerdemia is present which is also potent risk factor for CHD. The haemostatic measures are also abnormal favour hypercoaguable state in NIDDM specially microalbuminuric (Callier A, Rumtery A et al). This data also shows the increased free radical activity.
Both these abnormalities lead to endothelial cell dysfunction which may cause CHD in NIDDM population with microalbuminuria. After two year study (Mattock MB, Morish NJ, Liberti G er al) conclude that microalnuminuria is a significant risk maker for abnormality in NIDDM, independent of the other risk factors examined. Its presence can be regarded as an index of increased cardiovascular vulnerability and a signal for vigorous efforts at correlation of known risk factors.

Among men free of CHD at baseline, the independent risk factors for CHD morbidity and mortality were microalbuminuria, current smoking, high diastolic blood pressure, and high derum cholesterol. For the 100 NIDDM patients with microalbuminuria at baseline, the incidence of microalbuminuria was $29 \%$ over 7 year period. In that group, fasting plasma glucose, current smokingm preexisting CHD and high initial urinary albumin excretion rate were risk for development of microalbuminuria (all $\mathrm{P}<0.05$ ), when all women were analysed separately. Preexisting CHD was a significant risk factor in men only. (MB Mattock, DJ Barnes, Giviberti, Hkeen D Burt, JM hughes, AP Fizgerold B Sandhu and PG Jackson).

\section{Aims and Objectives}

In India due to high incidence of diabetes, mostly (98\%) NDMM (Chandalia HB/1995) the prevention from its complication is the risk, mostly coronary heart disease leads to death in NIDDM population. This work attempts to analyse the association of microalbuminuria and coronary heart disease in NIDDM population (type 2 diabetes).

$\checkmark$ To select cases of diabetes from diabetic OPD.

$\checkmark$ Micral test was done on every patient with diabetic history of five years or more.

$\checkmark$ Follow up of both group diabetes mellitus with microalbuminuria and diabetes mellitus without microalbuminuria. 
$\checkmark$ In follow up cardiovascular status of both group taken.

$\checkmark$ To conclude the study on microalnuminuria and its association with cardiovascular complication in type-2 diabetic patients.

Objective of this is to know the prediction power of microalbumiuria of detection of cardiovascular complication in NIDDM population (type 2 diabetics).

\section{Material and Methods}

The present study was carried out with type- 2 diabetic patients attending in diabetes clinic medical emergency and medical ward (General Medicine) of Patna Medical College \& Hospital, Patna, Bihar.

\section{Patients:}

Patients are randomize selected from outdoor, department and medical ward having

1) History of diabetes mellitus more than 5 years

2) Age $>45$ years

Reason for exclusion from the study include-

1) Pregnancy

2) Known case of diabetic nephropathy

3) Having infection

4) New diabetic renal disease

5) Evidence of urinary tract infection

6) Presence of other condition responsible for proteinuria e.g- Congestive heart failure

7) Other factors impairing the ability of patients to participate in the study

Patients are selected randomly who are attending Medicine O P D Most of these patients are referred from other centre because uncontrolled diabetes mellitus department. Hypertension is not our inclusion criteria but maximum of them are hypertensive.

Hypertension was diagnosed on the basis of JNCVIII recommendation. According to this systolic blood pressure over $150 \mathrm{mmHg}$ and diastolic blood pressure $90 \mathrm{mmHg}$ in patients over 60 years and systolic blood pressure of $140 \mathrm{mmHg}$ and diastolic blood pressure of $90 \mathrm{MMHg}$ everybody else is considered as hypertension. (Sources: JNC VIII, 2013).

All the patients were considered hypertensive as either their blood pressure satisfied the above criteria or were already being treated for hypertension. We randomly selected 117 patients (type 2 diabetes) first time appearing in our up patients department within 2 year of study (Dec. 2018 to November2020).

All patients were subjected to thorough clinical examination and necessary investigation and values were recorded. The patients were also questioned to ensure that they have made no modification in their diet or physical habit in recent past. In our selected patients very few had habits of cigarette smoking. cigarette smokers are discarded from our study. We also discarded patients having history of hypertension for more than two years. All patients underwent fomicral test for the detection of microalbuminuria. They are divided into two groups. Group A $(\mathrm{N} 0=86)$ Normoalbuminuric and gropu $\mathrm{B} \quad(\mathrm{N} 0=31)$ microalbuminuric.

Electrocardiography was done in all the patients and to see involvement of cardiovascularsystem.

\section{Blood Pressure Measurement}

Blood Pressure was measured with a standard mercury sphygmonometer with the patients lying down. Patients had taken rest for half an hour before measurement and instructed not to take coffee or cigarette within one hour before measurement. The cuff was applied to left arm for 15 minutes, after which the pressure was recorded two times at 5 minutes intervals, while the patients remained at rest. Diastolic blood pressure was recorded at the disappearance of the KOrotkoff sounds. The mean of two readings different by no more than $10 \mathrm{~mm}$ of $\mathrm{Hg}$ were recorded.

\section{Laboratory Test:}

Laboratory test includes:-

- Hemoglobin estimation by acid haematin method 
- Total and differential cont of leucocytes by visual microscopic method.

- Blood sugar estimation

Fasting 2 hour post parandial.

- Glycatedhemoglobin estimation

- Blood urea estimation

- Serum creatinine estimation

- $\quad$ Lipid profile

$$
\begin{aligned}
& >\text { Totalcholesterol } \\
& >\text { HDL } \\
& >\text { LDL } \\
& >\text { Triglyceride }
\end{aligned}
$$

- Urine examination- Which included.
a) Urine sugar by Benedict's test. All the patients of our study having possible test.so it not mention in our master chart.
b) Microscopic examination of urine for WBC, $\mathrm{RBC}$
c) Urine culture
d) Micral test.

\section{Micral Test:}

Micral test was done by micral test II of Boehringer Mennheim.

Principle: Test strip contains- Monoclonal antibodies against human albumin (Immunoglobin G) labeled with colloidal gold conjugate which specifically binds to urine albumin. Excess conjugate is retained in a separation zone containing immobilized human albumin so that only the albumin loaded gold conjugate reaches the detection zone. Coloured produced (while to red) is directly related to the albumin content of the urine. Cross reaction with other human proteins have been found to be $<0.5 \%$.

\section{Sample Material}

We collected the 12 hour overnight urine in a clean container.

Patients are told to take rest and not do physical exercise. All drugs are stopped 2 days before examination. The liquid intake is 1.5 to 2.0 liter average per day.

Morning sample of urine was taken in another clean container. The container was not washed with strongly oxidizing material. The container is thoroughly washed with water. The morning sample was tested immediately or if not possible stored in refrigerator in +2 to $8^{0} \mathrm{C}$ - the test was done on same day.

\section{Interpretation}

Reaction colour lighter than the color black corresponding to approx $30 \mathrm{mg} / \mathrm{L}$ albumin or more.

The specificity and sensitivity of micral test for the detection of microalbuminuria very accurately if we exclude the physiological factors and the pathological factors which gives the false positive results.

In a previous study showed that a spot urinary albumin concentration is of value in identifying or progression to nephropathy, and is simple to obtain at clinic. (Beatly UL, Ritdrie CM, Bull PM et al Diabetic Med-2004).

In another study which compare the double dipstick with nephlometric method showed and sensitivity of $90.8 \%$ and specificity of $80.1 \%$. The false positive results were associated with elevated urinary volume.

(Le Floch JP, Charles MA. Phillipon C; per emreter L Diabetic Med 1994)

\section{Electrocardiogram}

Electrocardiogram done in outpatient department with standard method. All the tewlve leads was done and ECG kept in record for analysis.

\section{Result}

The result of present study is to determine the microalbuminuria as a maker for the early detection of cardiovascular complication.

Two year long randomized study was done in OPD in Patna Medical College \& Hospital, Patna Bihar, in this study 117 patients were randomly selected of history of diabetes more than five years and age more than 45 years. We examined these patients and prescribed for micral test and other investigation. Continuous three morning sample were obtained and patients was diagnosed microalbuminuric when two out of three sample was positive. 
The study shows that microalbuminuria is independent marker of ischemic heart disease and cardiovascular complication in type 2 diabetic population.

Micral test is very cheap and can be done in outpatient department.

By diagnosing microalbuminuria, diabetic patients can be assessed in line of nephropathic complication as well as cardiovascular complication.

The cause of higher incidence of cardiovascular complication as well as cardiovascular complication in microalbuminuria patients may be genetic linked or other factors associated with it, like plasmingogen activator inhibitor insulin like molecules.

There is no realationship of microalbuminuria and other factor associated with ischemic heart disease in normal population like dyslipidaemia, hypertension and age. It is also not related with duration of diabetes to produce the complication.

\section{Conclusion}

We conclude that microalbuminuria is the independent marker for the cardiovascular complication in type 2 diabetes mellitus. This can be diagnosed by cheap and easy procedure "micral test".

Every type 2 diabetic's patients above the age of 45 irrespective of duration of diabetes should be examined for microalnuminuria and if positive should be go for test to diagnosed cardiovascular complication like treat mill test.

The larger study is necessary for the diagnosis and define role of microalbuminuria and also genetic and Biochemical profile like plasminogen activationg inhibitor and insulin like molecules should be exposed for the early diagnosis and its relation with microalbuminura.

\section{References}

1. Abnormal albuminura as a predictor of mortality and renal impairment in Chines patients with NIDDM. (Chan JC; Cheung
CK, Cheung MY; Swaminath R; Cockram CS;- Diabetic Care 2010)

2. Agarwal KK SinglaSweta $S$ et al : Prevalence of coronary riskfactor in type 2 diabetes without manifestation of overt coronary heart disease. J. Association Physiol. 2009; 57: 135-42.

3. Agarwal R.P Ola V, Bishnoi $\mathrm{P}$ et al, Prevalence of microalbuminuia and microalbuminuiacomplications and their risk factors in type 2 diabetes mellitus J AssoPhy Ind. $2014:$ : 62: 504-508.

4. Albuminuria and poor glycemic control predict mirtality in NIDDM. (Gall MA; Borch Johsen K; Hougarrd P; Nielsen FS; Parving HH; -Diabetes 2009.

5. Das S, Mishra DP, Sahu PK et al study of hsCRPmicroalnuminuria with type 2 diabetes mellitus in india, Journal of diabetes mellitus 2012.2

6. Diurnal variation of blood pressures and microalbuminuria in essential hypertension. Am Hypertens 2015.

7. Hyperinsulenemicmicroalbuminuria- a new risk indicator for coronary heart disease. Kussisto J, coronary heart disease. Kusisto j, Mykkanen L, PyordicLaaksom. Circulation2013.

8. Hyperlipidemia during diabetes mellitus; Recent development. (Guerel B; Ziegler D; Drouin P; - Service de M. Med. Toul. Presse Med 2012.

9. Microalbuminuria- a major risk factor in type II DM Schmitz AMaeath M. Daibet Med. 2013.

10. Microalbuminuria : Prognostic and therapeutic implication in diabetes mellitus, (Gilbert RE; Cooper ME; Mcnally PG; O'Brian RC; Taft J; - Diabet Med 2015.

11. Sosale A, Prasana Kumar KM, Sadikot SM et al: Chronic complication in nearly diagnose patients with type 2 diabetes mellitus in India (CINDI). Indian Journal of Endocrinology and Metabolism. 2014; 18, 355-360. 